Utah State University

DigitalCommons@USU

Space Dynamics Lab Publications

Space Dynamics Lab

$1-1-1976$

\title{
Optical Radiation from the Atmosphere
}

Doran J. Baker

Utah State University

William R. Pendleton Jr.

Utah State University

Follow this and additional works at: https://digitalcommons.usu.edu/sdl_pubs

\section{Recommended Citation}

Baker, Doran J. and Pendleton, William R. Jr., "Optical Radiation from the Atmosphere" (1976). Space Dynamics Lab Publications. Paper 2.

https://digitalcommons.usu.edu/sdl_pubs/2

This Article is brought to you for free and open access by the Space Dynamics Lab at DigitalCommons@USU. It has been accepted for inclusion in Space Dynamics Lab Publications by an authorized administrator of DigitalCommons@USU. For more information, please contact digitalcommons@usu.edu.

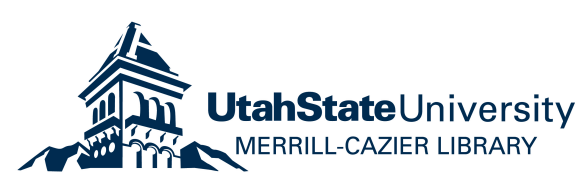


$91,50-62(1976)$

Baker, Doran J., and William R. Pendleton Jr. 1976. "Optical Radiation from the Atmosphere." Proceedings of SPIE 0091: 50-62. doi:10.1117/12.955071.

\section{OPTICAL RADIATION FROM THE ATMOSPHERE}

Doran J. Baker and William R. Pendleton, Jr.

Electro-Dynamics Laboratories, Utah State University

Logan, Utah 84322

\section{ABSTRACT}

The interface region which lies between the meteorological atmosphere of the Earth and "outer" space is a source of abundant optical radiation. The purpose of this paper is to provide the optical instrumentation engineer with a generalized understanding and a summary reference of naturally-occurring aerospace radiation phenomena. The colors of the radiation extend over the full optical spectrum from ultraviolet through the infrared. The emissions, observed during both day and night times, are rich in line and band spectra. The parameterization of atmospheric light by frequency (or photon energy) and by spectral radiance is discussed. The sources of the natural light from the gases of the atmosphere are grouped into four categories: (1) airglow mechanisms, (2) thermal processes, (3) scattering phenomena, and (4) auroral excitations. An overview of the characteristic spectral occurrences and intensities is given.

\section{INTRODUCTION}

The purpose of this paper is to provide a general understanding of the 1 ight which comes from the atmosphere of the Earth. This atmospheric radiation ranges from the far ultraviolet through the far infrared. We include exoatmospheric primary radiations such as sunlight, moonlight, starlight, planetary light, and zodiacal light, only to the extent that they are primary sources of subsequently radiated or scattered atmospheric light energy. For our purposes the definition of the upper limit of the atmosphere will be taken as the altitude at which molecules can leave the atmosphere without undergoing collisions along the way. In other words, the molecular mean-free-path approaches a scale height.

Figure 1 shows the range of the radiant energy spectrum interpreted in terms of the molecular physical phenomena involved. " We will consider the wavelengths of the 1 ight to range from about $1 \mathrm{~mm}$ for the far infrared to about $10 \mathrm{~nm}$ for the extreme ultraviolet. The categorizations of the subregions of the light spectrum have a high degree of arbitrariness and, of course, there are no abrupt frequency boundaries.

The visible light, which ranges from red through violet, involves energy transitions of the outer shell electrons of the atoms and molecules which make up the atmosphere. Ultraviolet light interactions can include photon transitions within the inner shells as well.

The infrared spectrum of radiation from the atmosphere, on the other hand, is dominated by energy mechanisms associated with the vibration of molecules. The mid-infrared region is rich with molecular fundamental rotation-vibration bands, whereas many of the overtones of the bands occur in the near infrared. Pure rotational spectra are seen in the far infrared.

\section{PARAMETERIZATION OF ATMOSPHERIC LIGHT}

The parameters of atmospheric light are frequency, radiance, and polarization. The frequency of light ranges from about $\frac{1}{4} \mathrm{THz}$ (terahertz) for the far infrared up to some 2,500 THz for the far ultraviolet. The prefix tera, we recal1, represents a factor of 1012 . Thus, the frequency of the optical radiation portion of the electromagnetic spectrum covers four orders.

\section{Frequency and Photon Energy}

Since light is emitted or absorbed in discrete energy quanta, the frequency of light is also characterized by specifying the photon energy,

$$
E \equiv h \nu \text {, }
$$

where ${ }^{2}$

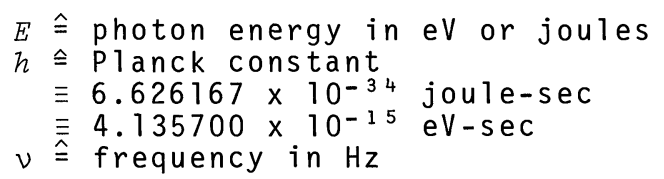


The photon energy ranges from about $10^{-3}$ eV for the far infrared to about 100 eV for the extreme ultraviolet.

The spectrum on the film of a grating spectrograph is almost linear with wavelength,

$$
\lambda \hat{=} v / \nu \equiv c / \eta \nu \text {, }
$$

where

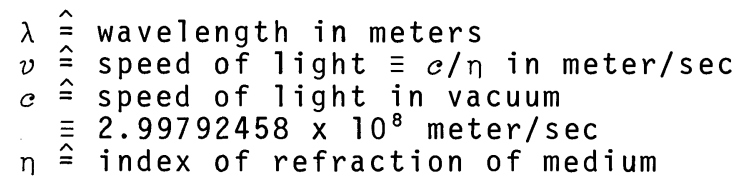

Hence, spectra of optical radiations are very commonly plotted with wavelength as the independent variable (abscissa). However, as shown explicitly in Equation (2), wavelength depends upon the index of refraction of the gas in the spectrograph. Commoniy used units of measure for the wavelengths of light are

$$
\begin{aligned}
& \text { micrometer }(\mu \mathrm{m}) \equiv 10^{-6} \text { meter } \\
& \text { nanometer }(\mathrm{nm}) \equiv 10^{-9} \text { meter } \equiv 10 \AA \\
& \text { angstrom }(\AA) \equiv 10^{-10} \text { meter } \equiv 10^{-4} \mu \mathrm{m}
\end{aligned}
$$

Unlike the grating spectrograph, the spectrum from a prism spectrograph departs markedly from linearity with wavelength. It is, in fact, more nearly linear with inverse wavelength, i.e., with wavenumber,

$$
\sigma \hat{=} 1 / \lambda \equiv \nu / v \equiv \eta \nu / c
$$

where

$$
\sigma \hat{\cong} \text { wavenumber in meter }{ }^{-1} \text {. }
$$

Wavenumber, like wavelength, is dependent upon the index of refraction of the gas in the spectrograph.

Wavenumber, which is the number of complete wave cycles in a unit distance, is being used increasingly to characterize light spectra. The use originated because of the discovery by Hartley, Balmer, Rydberg and others of multiplicative relationships in spectra that depend upon reciprocal wavelength differences. In other words, wavenumber is proportional to photon energy,

$$
\sigma=\eta E / h c .
$$

Also, the computer-generated "readout" of an interferometer-spectrometer is linear in wavenumber.

The most commonly used unit for wavenumber is reciprocal centimeter $\left(\mathrm{cm}^{-1}\right)$. As used by spectroscopists in practice, the $\mathrm{cm}^{-1}$ unit can be interpreted as an alternate unit for photon energy or for "cycles-per-second" frequency. This is because they usually convert their wavenumber values to vacuum wavenumber using

$$
\sigma_{v a c}=\sigma_{a i r} / n_{a i r}
$$

which is simply

$$
\sigma_{v a c} \equiv v / c
$$

Thus, vacuum wavenumber is nothing more than frequency with a scale factor of $1 / c$, where one hertz is equivalent to $1 /\left(3 \times 10^{8}\right)$ meter-1 or $1 /\left(3 \times 10^{10}\right) \mathrm{cm}^{-1}$. In wavenumber units light ranges from about $10 \mathrm{~cm}^{-1}$ in the far infrared to $10^{7} \mathrm{~cm}^{-1}$ in the far ultraviolet. A memory prop is the following: "1 $\mu \mathrm{m}$ corresponds both to $10,000 \AA$ and to $10,000 \mathrm{~cm}-1$ and is equivalent to a photon energy of about one eV." (To be exact, it is 1.24 eV.)

It is important to note that the spectral resolution of a spectrometer expressed in wavenumber is related to that in wavelength by

$$
\Delta \sigma=-\Delta \lambda / \lambda^{2}
$$


where

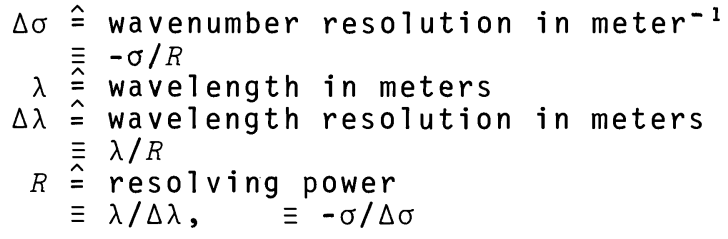

The negative sign in these incremental (differential) equations is used because increasing increments of $\sigma$ and $\lambda$ are opposite from one another. The definition of resolution element is taken as the interval between the half response points on either side of the peak response. ${ }^{3}$

The wavelength resolution element $\Delta \lambda$ of a monochromator is somewhat independent of $\lambda$. However, for the interferometer it is the resolution element $\Delta \sigma$ that is constant with wavenumber.

\section{Intensity}

Another parameter by which the atmospheric light is characterized is the intensity. Generally atmospheric sources are extended, i.e., they have significant spatial extent in contrast with the point sources common to astronomy. The light intensity is specified by the radiance

$$
L \hat{=} \lim \Delta \Phi / \Delta S \Delta \Omega
$$

where

$$
\begin{aligned}
L & \hat{=} \text { radiance in watt } \mathrm{m}^{-2} \mathrm{sr}^{-1} \\
\Delta \Phi & \hat{=} \text { incremental light flux in watts } \\
\Delta S & \hat{\equiv} \text { incremental apparent surface area in meter }{ }^{2} \\
\Delta \Omega & \hat{=} \text { incremental solid angle in steradians }
\end{aligned}
$$

If the flux $\Phi$ is expressed in lumens, this extended source entity is called brightness. Thus, the radiance or brightness of a source is the light flux emitted per unit area of the source into each unit of solid angle "away from the source."

Radiance can be converted from power flux to photon flux units using the relationship

$$
\begin{aligned}
1 \text { photon/sec } & \equiv 1.986475(1 / \lambda) \times 10^{-25} \text { watt } \\
& \cong 2 \times 10^{-25} / \lambda \text { watt. }
\end{aligned}
$$

Another unit for the photon radiance which has been widely adopted by atmospheric scientists is the rayleigh defined by ${ }^{4}$

$$
1 \text { rayleigh } \hat{=}(1 / 4 \pi) \times 10^{10} \text { photons } \sec ^{-1} \mathrm{~m}^{-2} \mathrm{sr}^{-1}
$$

The Baker formula for conversion from power radiance $L$ to rayleigh (photon) radiance $R$ is

$$
R=2 \pi \lambda L \times 10^{15},
$$

where $L$ is in watt $\mathrm{m}^{-2} s \mathrm{r}^{-1}$ and $\lambda$ is in meters.

The light flux into a photometer which remotely views an extended source of radiance $L$ is easily computed. Due to an equivalence theorem, ${ }^{5}$ if the source can be assumed to uniformly fill the viewing field of the instrument, then

$$
\Phi=L A \Omega,
$$

where

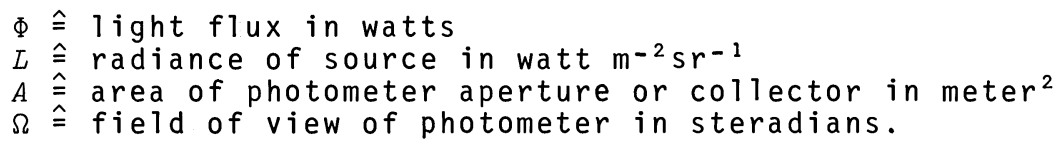

The ordinate of a spectrum is used to indicate the intensity of the light at the various wavelengths or wavenumbers. This power spectral density or spectral radiance,

$$
L_{\lambda} \hat{=} \lim \Delta L / \Delta \lambda
$$


or

$$
L_{\sigma} \hat{=} \lim \Delta L / \Delta \sigma
$$

is derived from the wavelength or wavenumber resolution of the spectrometer and is generally expressed on a per angstrom, per nanometer, per micrometer or on a per reciprocal centimeter basis. For example, photon spectral radiance is often expressed in rayleighs per angstrom or rayleighs per reciprocal centimeter.

The rayleigh is a very useful unit of measure for atmospheric light. Features of the airglow generally are expressed in rayleighs (R), those of aurora in kilorayleighs (kR), and those of thermal and scattered radiation in megarayleighs (MR).

\section{Light Polarization}

Any incident beam of light can be specified in terms of two components that are polarized in perpendicular azimuths. The emission from a particular molecule has one polarization component with the direction specified by the orientation of the electric field vector. However, an ensemble of molecules will have the azimuths of the oscillators randomly distributed and thus the net light will be unpolarized. Elliptical polarization will result from subsequent scattering and reflections. The degree of polarization is given by

$$
\beta \hat{=}\left(L_{\max }-L_{\min }\right) /\left(L_{\max }+L_{\min }\right)
$$

where

$$
\begin{aligned}
& L_{\max } \hat{=} \text { maximum radiance observed when a polarizer is rotated } \\
& L_{\min } \hat{=} \text { minimum radiance observed when a polarizer is rotated } \\
& \text { in the beam }
\end{aligned}
$$

When $\beta=1$ the beam is said to be plane polarized; when $\beta=0$ the light is unpolarized (or circularly polarized).

\section{SOURCES OF ATMOSPHERIC LIGHT}

The natural light from the atmosphere can be categorized into four general areas according to the source processes. These categories are: (I) airglow, (II) thermal radiation, (III) scattered light, and (IV) aurora. Due to the interactive nature of the atmospheric mechanisms involved, exclusive definitions are inappropriate; however, descriptive categories are helpful for a general overview.

There are six principal emission mechanisms leading to atmospheric radiation which we will discuss in terms of the preceding source categories:

1. Chemiluminescence- emission due to the excitation of one or more of the products of exothermic chemical reactions.

2. Fluorescence- absorption of photons of one energy with subsequent emission of photons of lesser energy.

3. Energy transfer-emission due to the excitation of one particle transferred from another particle during a collision.

4. Resonance scattering-absorption and emission of photons without a change in the photon energy.

5. Charged particle collisions-emission due to the excitation of atoms or molecules by fast charged particles.

6. Dissociative recombination-emission due to the excitation of atoms or molecules in the recombination of thermal ions and electrons or by ion-ion recombination.

\section{Airglow}

The night airglow, discovered by Newcomb in 1901,6 is the relatively faint nonthermal luminescence that results from photochemical reactions of the dilute gases of the upper atmosphere. The airglow is basically produced by sunlight; it originates during the daytime and persists throughout the night. Solar ultraviolet energy is stored at altitudes around and above the mesopause in dissociated, ionized and excited species during the day and then released at night by various relaxation processes such as chemical association, transfer of excitation and ionic recombination (emission mechanisms 1, 3 and 6). The airglow "mirrors" the chemical state of the upper atmosphere, making it possible to remotely monitor it in astonishing detail. 
Since the photochemical processes that cause the airglow depend critically upon the conditions of illumination, there are marked differences between the daytime and the nighttime airglow. There are also features peculiar to the twilight airglow, the transition between day and night airglow. When the upper atmosphere is illuminated by sunlight, it is impossible to completely distinguish scattering from the other airglow processes. Resonance scattering from certain species is therefore often included as part of the daytime and twilight airglow.

The most intense night airglow originates in a layer near the mesopause. This bright band can easily be seen when viewed edge-on from rockets or satellites (Fig. 2 ). However, some airglow features are most prevalent at higher altitudes, notably atomic hydrogen Lyman alpha, the atomic oxygen red line and nitric oxide infrared bands.

Detailed spectral atlases of the atmospheric night airglow may be found in the literature. Unattenuated radiance values for the brightest lines and bands are summarized in Table 1 for typical cases of nighttime, twilight and daytime conditions. Measurements are usually referred to the zenith. The radiance generally increases away from the zenith as

$$
R=R_{z} \sec \theta \text {, }
$$

where

$$
\begin{aligned}
R & =\text { photon radiance in rayleighs at angle } \theta \\
R_{Z} & =\text { zenith photon radiance in rayleighs } \\
\theta & =\text { angle from zenith in radians }
\end{aligned}
$$

This is the van Rhijn effect ${ }^{7}$ which is seen when an optically-thin airglow layer is viewed obliquely.

The first airglow feature discovered ${ }^{8}$ was the green line of atomic oxygen at $5577 \AA$ which constitutes about $7 \%$ of the clear, moonless nighttime atmospheric light seen by a darkadapted eye. About $15 \%$ of the light is attributed to zodiacal 1 ight, which is sunlight scattered by interplanetary dust. The remainder of the natural visual light of the night sky is due to other airglow species $(33 \%)$, to starlight and to other scattered light.

The 5577- $\AA$ green 1 ine at low altitudes in the night airglow results from a chemical reaction, namely, the Chapman reaction ${ }^{9}$

$$
\begin{gathered}
0+0+0 \rightarrow 0_{2}+0^{*} \\
0^{\star} \rightarrow 0+h \nu
\end{gathered}
$$

where the asterisk (*) denotes an electronically-excited state. The characteristic shape of the altitude profile in which the airglow forms is called a chapman Zayer. ${ }^{0}$

A pair of relatively bright atomic oxygen lines occur at 6300 and $6364 \AA$. This oxygen red multiplet airglow originates within the F-region of the ionosphere and is attributed to dissociative recombination with photoelectronsil

$$
\begin{gathered}
0_{2}^{+}+e^{-} \rightarrow 0^{*}+0 \\
0^{*} \rightarrow 0+h \nu
\end{gathered}
$$

Airglow radiations from nitrogen, the most abundant gas of the terrestrial atmosphere, are sparse except for some atomic lines. The neutral nitrogen molecule in the electronic ground state has no dipole moment and therefore is a poor radiator. The most intense night airglow comes from hydroxyl molecules at an altitude of about $35 \mathrm{~km}$. The chemical reaction

$$
\begin{gathered}
\mathrm{H}+\mathrm{O}_{3} \rightarrow \mathrm{OH}^{\ddagger}+\mathrm{O}_{2} \\
\mathrm{OH}^{\ddagger} \rightarrow \mathrm{OH}+h \nu
\end{gathered}
$$

gives rise to these Meinel rotation-vibration bands of radiation which are widely distributed throughout the visible and near infrared portions of the spectrum. The symbol ( $\neq$ ) denotes that the hydroxyl radical is in a vibrationally-excited state.

Excited oxygen molecules are formed by a three-body reaction in which atomic oxygen recombines,

$$
\begin{gathered}
0+0+\mathrm{N}_{2} \rightarrow 0_{2}^{*}+\mathrm{N}_{2} \\
0_{2}^{*} \rightarrow \mathrm{O}_{2}+h v
\end{gathered}
$$


This gives rise to the Herzberg electronic bands in the ultraviolet and to the atmospheric bands in the red and near infrared spectral regions. The molecular oxygen emissions are the brightest luminescent features of the daytime airglow. The emission layer peaks at about $60 \mathrm{~km}$, with two important infrared bands occurring at 1.27 and $1.58 \mu \mathrm{m}$. A transfer of excitation mechanism that is important at about $100 \mathrm{~km}$ in the airglow is ${ }^{13}$

$$
\begin{gathered}
0^{*}+0_{2} \rightarrow 0+0_{2}^{*} \\
0_{2}^{*} \rightarrow 0_{2}+h v
\end{gathered}
$$

which gives rise to bands at 7619 and $8640 \AA$.

Fluorescence is an important process in exciting some emissions in the daytime and twilight airglow. ${ }^{4}$ Also, resonance scattering by upper-atmospheric gas species is often included as part of a listing of daytime airglow spectra. Such features are the atomic hydrogen Lyman- $\alpha$ ine at $1216 \AA$, the sodium multiplet at 5890 and $5896 \AA$, helium at $10,830 \AA$ and the NO $\gamma$ bands in the ultraviolet.

Thermal Radiation

The process called thermal emission is one in which kinetic energy of the atmospheric constituents is transferred to the radiation field. This matter-radiation interaction is the reverse of absorption. Thermal emission processes constitute a part of the theory of radiative transfer which involves the energy distribution of the qas molecules. This energy is possessed in four forms: translational, electronic, vibrational, and rotational. Since the last three forms are quantized, they take part directly in the exchange of energy from matter to the radiation field. Energy transfer between the four modes takes place during collisions. As there is an adjustment upon every collision, the translational energies of the molecule can rapidly relax to Boltzmann equilibrium.

In equilibrium the ratio of the number density of molecules which possess an energy $E_{1}$ relative to the density of those with energy $E_{2}$ is described by an exponential Boltzmann distribution,

$$
\frac{n\left(E_{1}\right)}{n\left(E_{2}\right)}=\frac{g_{1}}{g_{2}} e^{\left(E_{2}-E_{1}\right) / k T}
$$

where

$$
\begin{aligned}
& n \hat{=} \text { number density of molecules in } \mathrm{m}^{-3} \\
& g \hat{=} \text { statistical weight of state } \\
& E \hat{=} \text { energy of state in joules } \\
& k \hat{=} \text { Boltzmann constant } \\
& \equiv 1.38047410 \times 10^{-23} \text { joule/ }{ }^{\circ} \mathrm{K} \\
& T \stackrel{\hat{=}}{=} \text { absolute temperature in }{ }^{\circ} \mathrm{K}
\end{aligned}
$$

Thus, where $E$ represents the kinetic energy, Equation (22) leads to the definition of a kinetic temperature even in the absence of total thermodynamic equilibrium. Similarly, equilibrium within a restricted group of energy levels leads to a rotational temperature or vibrational temperature. The atmosphere can be ascribed a kinetic temperature (by definition) at altitudes below the exosphere. Airglow species are often in rotational equilibrium but at the higher altitudes are seldom in vibrational equilibrium.

The substantial emission observed from the atmosphere through much of the infrared region is due to (1) the presence of polyatomic molecules having strong rotation and vibrationrotation bands and (2) the fact that at atmospheric temperatures thermal processes can excite rotational and vibrational transitions. ${ }^{15}$

Thermal emissions from the atmosphere are very bright in the near and mid-infrared regions. The emissivity of air is high at the wavelengths where the characteristic bands of $\mathrm{H}_{2} \mathrm{O}, \mathrm{CO}_{2}$ and $\mathrm{O}_{3}$ occur, such as $2.7,4.3,6.3,9.6$, and $15 . \mu \mathrm{m}$. The major features of typical spectra of atmospheric thermal radiations are summarized in Table 1 . These thermal emitters include methane $\left(\mathrm{CH}_{4}\right)$, nitric acid $\left(\mathrm{HNO}_{3}\right)$, the nitric oxides (NO, $\left.\mathrm{NO}_{2}\right)$, and $0(63 \mu \mathrm{m})$.

Because of the temperature and concentration dependence, the thermal emissions are markedly dependent upon altitude. For instance, nearly all of the water vapor emission originat es below $5 \mathrm{~km}$. The zenith spectral radiance typically drops two orders of magnitude from sea level to $10 \mathrm{~km}$ and then another tenfold between 10 and $30 \mathrm{~km}$.

The lower atmosphere is opticalzy-thick to most of the thermal radiations, as distinguished from opticalzy-thin conditions for most of the airglow emissions. Radiative energy is transported by the complicated absorption-reemission process with the e-fold attenuation 
length being less than several kilometers. Optical depth is given by

$$
D_{0}-D=\ln L_{\lambda} / L_{\lambda O}
$$

where

$$
\begin{aligned}
D= & \text { optical depth in meters } \\
L_{\lambda}= & \text { spectral radiance in watts } \mathrm{m}^{-2} \mathrm{sr}^{-1} \mu \mathrm{m}^{-1} \text { at one location } \\
& \text { relative to that at position " } \mathrm{ol}^{-1}
\end{aligned}
$$

At altitudes below about $70 \mathrm{~km}$ the optically-thick condition prevails for many of the thermal species. The good radiators are good absorbers. Below this altitude the time for deactivation by collision is typically less than the radiative lifetime. Local thermodynamic equilibrium can then generally be assumed. Due to the infrared opacity and the large solid angle subtended by the surface, earthshine is an important source of infrared energy to the atmiosphere below the mesopause.

\section{Scattered Light}

Looking overhead, a clear daytime sky is some 10 million times brighter than the night sky. This is, of course, due to scattering of sunlight. Scattering of light in the atmosphere may be grouped into three categories:
(1) resonant scattering by atoms and molecules
(2) Rayleigh scattering by atoms and molecules
(3) Mie scattering by aerosols.

Resonant scattering occurs when the incident photons have a frequency close to that of a possible energy transition in one of the gas atoms or molecules. The interacting quantum causes a transition to a higher excited state having a short lifetime. The transition to the lower state takes place in one step, and the process is called coherent scattering. The resonant-scattering processes generally involve ground-state connected transitions.

For molecules which have only narrow states of internal energy and incident photons which have a frequency far from that of a possible transition, a "simple" scattering model can be used for gas molecules. This Rayleigh theory of molecular scattering gives a scattering coefficient that is proportional to the fourth-power of the frequency, 16

$$
s_{R} \propto \nu^{4} \propto 1 / \lambda^{4} .
$$

Thus, in exceptionally clear weather the spectral brightness distribution of the daytime sky differs from the solar spectrum in a general way by a Rayleigh factor which is proportional to $\lambda^{-4}$. In turbid atmosphere the proportionality is $v^{n}$ where $n$ is generally 1 ess than 4.

A typical daytime spectrum of the sky is very complicated. The actual color of the sky depends both upon the solar zenith angle and the direction of the line of sight. Rayleigh scattering, multiple scattering, selective attenuation of sunlight by the atmospheric gases, and scattered earth albedo all must be taken into account. Spatially, the radiance varies about one order of magnitude over the daytime sky. It is brightest in the aureole around the sun and at the horizon; the minimum occurs relatively near the zenith on the solar meridian opposite the sun.

The Rayleigh-scattered light has a high degree of optical polarization. The maximum polarization occurs on the solar meridian at a point $90^{\circ}$ away from the sun. The degree of polarization (Eq. 15) of the sky generally lies between 0.5 and 0.7 and does not exceed about 0.85 even on an exceptionaliy clear day with the sun at the horizon. There are three angles along the meridian where the polarization drops to zero. ${ }^{7}$

Scattered moonlight resembles sunlight but is very much weaker. For a full moon at night the sky brightness is about $2 \times 10^{-6}$ that of solar daytime; for a new moon the ratio drops to $10^{-9}$. The sky brightness due to a high full moon corresponds to that of solar twilight at a solar depression angle. (below the horizon) of about $10^{\circ}$. The night sky brightness due to the sun is significant to a solar depression angle of about $18^{\circ}$. The contribution due to a full moon can be neglected at a depression angle of greater than about $7^{\circ}$.

The scattering of light from "large" particles ( $>0.1 \mu \mathrm{m}$ ) is modeled by the complicated Mie theory. In fact, the Rayleigh theory is the smali particle special case of the Mie theory. 16 In general, the scattering from the large particles varies as $v^{2}$, $i . e$., the scattering is "whiter." The scattering coefficient is less than for the small particles, and there is a strong forward component. These so-called aerosol particles are water droplets, ice crystals, dust, microorganisms, etc., and are a very active optical component of the atmosphere (rainbows, aureoles, haloes, etc.). Most of the aerosols are concentrated 
in the height range below about $4 \mathrm{~km}$, but high-altitude aerosols are also present and give rise to phenomena such as the noctilucent clouds which occur at about 75 km. These 1 uminous clouds make their appearance between the twilight arch at the horizon and the darkening night sky overhead.

The zodiacal light is exoatmospheric scattered sunlight due to interplanetary dust. The gegenschein is particularly bright zodiacal light that is seen at the antisolar direction.

\section{Aurora}

The aurora borealis in the north and the aurora australis in the south generally appear as faint luminous phenomena during the night. However, spectacular displays may be seen at times in certain locations free from scattered city lights. The auroras appear in the sky most frequently in two zones around the magnetic poles of the Earth. A wide range of spatial forms and structures are seen. General auroral form categories are: (1) relatively stable bands or arcs, (2) rays, (3) irregular patches, and (4) large homogeneous areas.

The auroral light results from the injection of fast, charged particles, principally solar electrons and protons, into the Earth's atmosphere. ${ }^{8}$ The first spectral studies of the aurora were made by Angstrom in 1867.20 The optical spectrum is characterized by numerous emission lines and bands from neutral and singly ionized atomic and molecular nitrogen and oxygen.21 Hydrogen emission lines are regular features of proton auroras; sodium and helium lines are occasionally observed. Table 3 summarizes the character of auroral emission species.

The most frequently observed auroral emissions are the atomic oxygen auroral green 1 ine at $\lambda 5577$ and the red line multiplet at $\lambda 6300 / 64$. The excitation of the green line is by direct electron excitation of 0 , electron dissociation of $0_{2}$ and by energy transfer from $\mathrm{N}_{2}^{*}$. The altitude of the emission is generally between 100 and $160 \mathrm{~km}$ where the energy deposition from the auroral particles takes place. The oxygen excitation state which results in the red line is long-lived $(110 \mathrm{sec})$ and is de-excited by $\mathrm{N}_{2}$; therefore the red aurora originates from high altitudes. The mechanisms involve transfer from $N^{*}$, secondary electrons (< 250 $\mathrm{km})$ and thermal electrons $(>250 \mathrm{~km})$. The radiance of the $0(5577 \AA)$ line is used as the standard for categorizing the auroral intensity. The International Brightness Coefficient (IBC) 22 of an aurora is called I, II, III, or IV according to whether the photon radiance of the green line in kilorayleighs is 1 , 10, 100, or 1000, respectively.

The ionization of molecular nitrogen leaves many of the molecular ions in the upper state of the first negative bands,

$$
\begin{aligned}
\mathrm{N}_{2}+e^{-} \rightarrow \mathrm{N}_{2}^{+*}+e^{-}+e^{-} \\
\mathrm{N}_{2}^{+\star} \rightarrow \mathrm{N}_{2}^{+}+h \nu
\end{aligned}
$$

This leads to emission in bands including the 3914,4278 and $4709 \AA$. The ratio between the total rate of ionization and the emission of $\lambda 3914$ photons is 18 at $100 \mathrm{~km}$. Due to the promptness with which the radiation is emitted, either the $\mathrm{N}_{2}^{+}$first negative $\lambda 3914$ or the $\lambda 4278$ band is an even better auroral diagnostic monitor than is the relatively slowly-emitted $\lambda 5577$ green 1 ine.

Emissions of neutral molecular nitrogen in the Vegard-Kaplan (ultraviolet), first positive (red-infrared), and second positive (violet-ultraviolet) bands result from excitation by secondary electrons ${ }^{2} 3$

$$
\begin{gathered}
\mathrm{N}_{2}+e^{-} \rightarrow \mathrm{N}_{2}^{\star}+e^{-} \\
\mathrm{N}_{2}^{\star} \rightarrow \mathrm{N}_{2}+h v
\end{gathered}
$$

Secondary electrons also produce excited states of molecular oxygen which can radiate in the red and infrared ${ }^{24}$

$$
\begin{gathered}
0_{2}+e^{-} \rightarrow 0_{2}^{\star}+e^{-} \\
0_{2}^{\star} \rightarrow 0_{2}+h v
\end{gathered}
$$

Balmer lines result from collisional excitation of auroral $H$ and also charge exchange, e.g.,

$$
\begin{gathered}
\mathrm{H}^{+}+\mathrm{N}_{2} \rightarrow \mathrm{H}^{*}+\mathrm{N}_{2}^{+} \\
\mathrm{H}^{*} \rightarrow \mathrm{H}+h \nu
\end{gathered}
$$

The emission 1 ines are $H \alpha$ at $6563 \AA$ and $H \beta$ at $4861 \AA$. 


\section{SPECTRAL ATLASES}

A number of good spectral atlases of atmospheric emission features are available in the literature. For airglow the reader is referred to the reviews and atlases of Chamberlain 18 and of Krassovsky et $a z^{2}{ }^{2}$ Additional visible spectral references are by Ingham and Broadfoot and Kendal1.27 In the infrared the altas of Vallance Jones 3 and the spectra of Connes and Gush, 28 Gush and Buijs, 29 Baker et al.,24 Bunn and Gush, 30 and Murcray ${ }^{3}$ are helpful. Table 1 gives a generalized working estimate summary of atmospheric radiation.

The authors are grateful for the assistance of Loretta Clyde, Kathy Bayn, Kathy Baker, Carla Lewis and Glen Allred. The review by Ralph Embry is also acknowledged.

\section{REFERENCES}

1. D.J. Baker and W.L. Brown, "Presentation of Spectra," App. Optics, Vo1. 5, 1966, p. 1333 .

2. E.R. Cohen and B.N. Taylor, J. Physical Chemical Reference Data, Vo1. 2, $1961, \mathrm{p} .715$.

3. J. Strong, Concepts of Classical Optics, Freeman, San Francisco, 1959, pp. $210-212$.

4. D.J. Baker and G.J. Romick, "Interpretation of the Unit 'Rayleigh' in Terms of Column Emission Rate or Apparent Radiance Expressed in International Systems IInits," App. Optics, Vol. 15, 1976 .

5. D.J. Baker, "Rayleigh, the Unit of Light Radiance," App. Optics, Vo1. 13, 1974, p.2160.

6. S. Newcomb, "An International Review of Spectrsocopy and Astronomical Physics," Astrophysics J., Vol. 14, 1901, p. 297.

7. P.J. van Rhijn, "On the Brightness of the Sky at Night and the Total Amount of Starlight," Astrophysics J., Vol. 50, p. 356.

8. J.W. Chamberlain, Physics of the Aurora and AirgZow, Academic Press, New York, 1961.

9. S. Chapman, "Some Phenomena of the Upper Atmosphere," (Bakerian Lecture). Proc. Roy. Soc., Vol. Al32, i931a, pp. 353-374.

10. S. Chapman, "Absorption and Dissociative or Ionizing Effects of Monochromatic Radiation in an Atmosphere on a Rotating Earth," Proc. Phys. Soc., Vol. 43, 1931b, pp. $26-45$.

11. D.R. Bates and H.S. Massey, "The Basic Reactions in the Upper Atmosphere. II. The Theory of Recombination in the Ionized Layers," Proc. Roy. Soc., Vo1. 192, 1947a, pp. 1-16.

12. D.R. Bates and M. Nicolet, "The Photochemistry of Atmospheric Water Vapor," J. Geophys. Res., Vol. 55, 1950b, pp. 301-327.

13. A. Vallance Jones, "The Infrared Spectrum of the Airglow," Space Sci. Rev., Vol. 15, 1973, pp. $355-400$.

14. D.M. Hunten, "Airglow-Introduction and Review," The Radiating Atmosphere, B.M.

McCormac (ed.), D. Reidel Publ. Co., Dordrecht, Holland, 1971 .

15. J.T. Houghton, "Infrared Emission from the Stratosphere and Mesosphere," Proc. Roy.

Soc., Vol. 288, 1965, p. 545.

í. R.M. Goody, Atmospheric Radiation, Clarendon Press, 0xford, 1964, p. 290.

17. G.V. Rozenberg, Twilight, A Study in Atmospheric Optics, Plenum Press, New York, 1966, p. 15 .

18. R.K. Soberman, "Noctilucent Clouds," Scientific American, Vol. 208, p. 50.

19. A. Omholt, The Optical Aurora, Springer-Verlag, Berlin, 1971.

20. S. Chapman, "History of Aurora Airglow, "Aurora and Airglow, Reinhold Publishing

Corp., New York, 1967 , p. 15.

2i. A. Vallance Jones, "Auroral Spectroscopy," Space Sci. Rev., Vo1. 11, 1971, p. 776.

22. M. Walt, Auroral Phenomena, Stanford University Press, Stanford, 1965, p. 40.

23. R.C. Whitten and I.G. Poppoff, Fundamentals of Aeronomy, Wiley, New York, 1971 , p.3.

24. D. Baker, A. Steed, R. Huppi and W. Pendleton, Jr., "Near Infrared Spectrum

of an Aurora," J. Geophys. Res, Vol. 81 , 1976 (in publication).

25. V.I. Krassovsky, N.N. Shefov and V.I. Yarin, "Atlas of the Airglow Spectrum 3000-

$12400 \AA$," Pianetary Space Sci., Vol. 9, 1962, pp. 883-915.

26. M.F. Ingham, "The Spectrum of the Airglow," Scientific American, Vo1. 226, 1972, pp. $78-85$.

27. A.L. Broadfoot and K.R. Kendall, "The Airglow Spectrum, 3100-10,000 А," J. Geophys. Res., Vol. 73, 1968, pp. 426-429.

28. J. Connes and H.P. Gush, "Spectroscopic du Ciel Nocturne dans l'Infrarouge par

Transformation de Fourier," J. Phys. Radium, Vol. 20, 1959, p. 915.

29. H.P. Gush and H.L. Buijs, "The Near Infrared Spectrum of the Night Airglow observed from High Altitude," Can. J. Phys., Vol. 42, 1964, p. 1037.

30. F.E. Bunn and H.P. Gush, "Śpectrum of the Airglow Between 3 and 4 Microns," Can. J.

Phys., Vol. 50, 1972, p. 213 (also "Between 4 and 8 Microns," Vol. 48, 1970, p.98).

$3 i$. D.G. Murcray et al., "Atomspheric Emission at High Altitudes," Final Report AFCRL-

72-0353, University of Denver, June 1972.

32. J. Hennes and L. Dunkleman, "Photographic Observations of Nightglow from Rockets,"

J. Geophys. Res., Vol. 71, 1966, p. 755. 


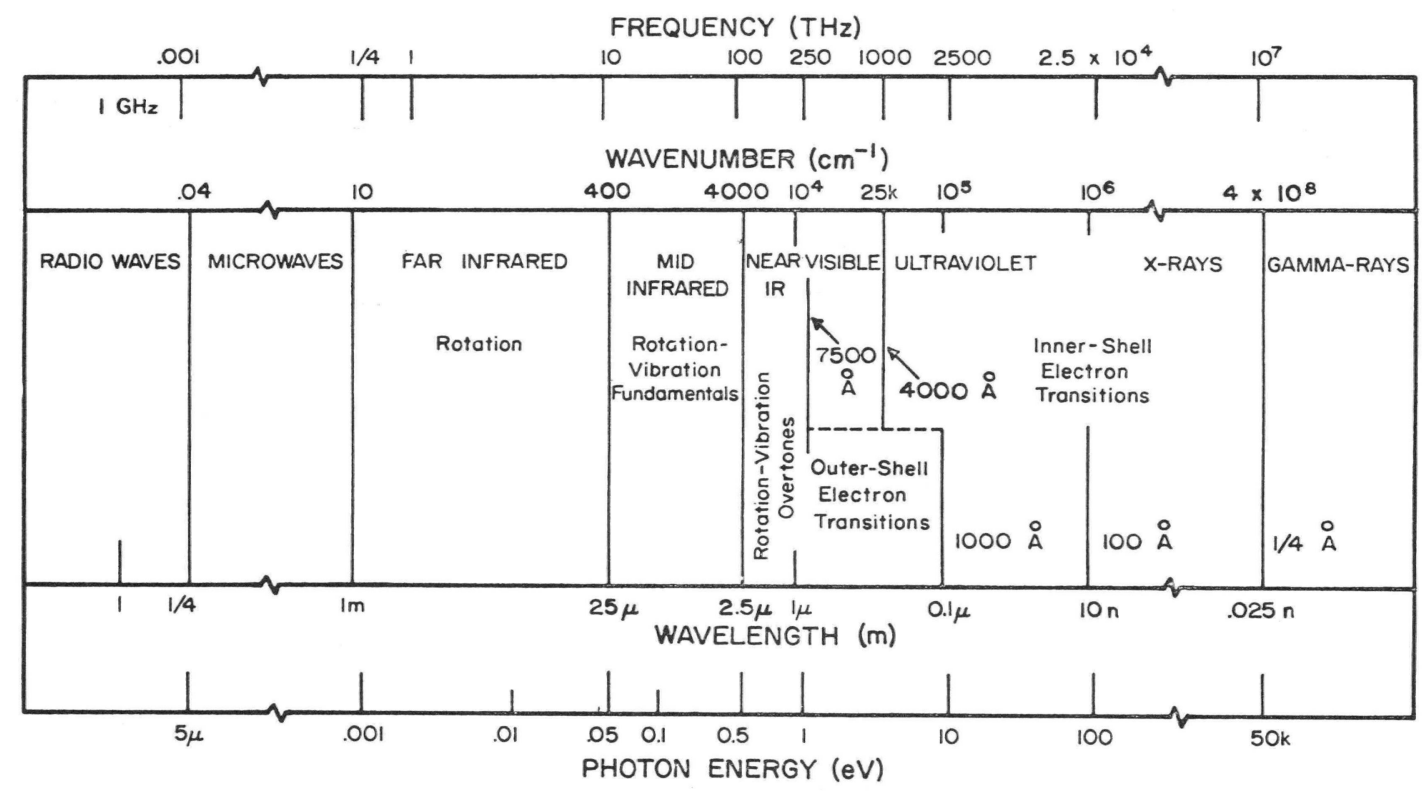

Fig. 1. Radiant energy spectrum interpreted in terms of gas emissions.

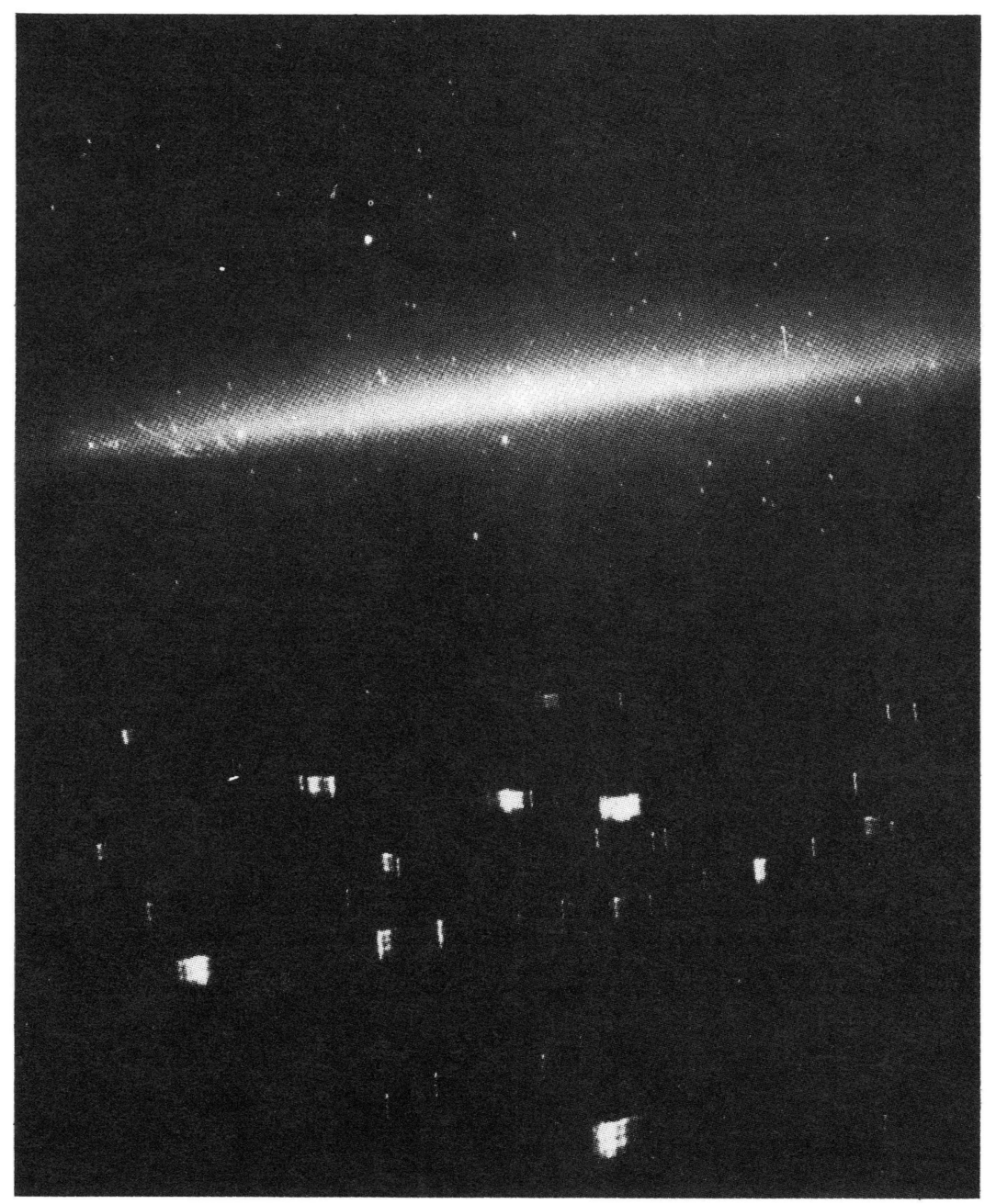

Fig. 2. Airglow layer seen edge-on from rocket at $93 \mathrm{~km} .{ }^{32}$ 
DORAN J. BAKER, WILLIAM R. PENDLETON, JR.

Table 1. Working-estimate summary of typical atmospheric radiation features.

\begin{tabular}{|c|c|c|c|c|c|c|}
\hline \multirow{2}{*}{$\begin{array}{c}\text { Wayelength } \\
(\AA \text { or } \mu \mathrm{m})\end{array}$} & \multirow{2}{*}{ Species } & \multirow{2}{*}{ Identification ${ }^{b}$} & \multicolumn{4}{|c|}{ Rayleigh Radiance ( $R$ ) in the Zenith } \\
\hline & & & Day & Twilight & Night & IBC III Aurora \\
\hline $304 \AA$ & $\mathrm{He}^{+}$ & $2^{2} p$ & $5-10\{750\}^{C}$ & & & \\
\hline 584 & $\mathrm{He}$ & $2 p^{1} p^{\circ}(2 u v)$ & $\frac{1}{2}-1 \quad k\{750\}$ & & $\approx 5$ & \\
\hline 834 & $0^{+}$ & $2 s 2 p^{4}{ }^{4} p(1 u v)$ & $0.5 k\{200\}$ & & $15\{\sim 130-215\}$ & $5 \mathrm{k}$ (est.) \\
\hline $955-1438$ & $\mathrm{~N}_{2}$ & $b^{1} \Pi_{U}(B-H)$ & & & & \\
\hline 989 & 0 & $3 s^{13} D^{\circ}(5 u v)$ & $\sim 7 k$ (est.) & & & \\
\hline 1026 & $\mathrm{H}$ & $3^{2} P\left(L_{\beta}\right)$ & & & $10\{200\}^{d}$ & \\
\hline 1027 & 0 & $3 d^{3} D^{\circ}(4 u v)$ & & & & \\
\hline 1085 & $\mathrm{~N}^{+}$ & $2 s 2 p^{3}{ }^{3} D^{\circ}(1 u v)$ & & & & $1 \mathrm{k}$ (est.) \\
\hline 1135 & $\mathrm{~N}$ & $2 s 2 p^{4}{ }^{4} p(2 u v)$ & & & & \\
\hline 1152 & 0 & $3 s^{1}{ }^{1} D^{\circ}(6 u v)$ & $\sim 1 \mathrm{k}$ (est.) & & & \\
\hline 1200 & $\mathrm{~N}$ & $3 s^{4} P(1 u v)$ & $0.4\{180\}$ & & & $5 k$ \\
\hline 1216 & $\mathrm{H}$ & $2^{2} P\left(L_{\alpha}\right)$ & $14 k\{1000\}$ & & $4 \mathrm{k}\left[10^{2}-10^{5}\right]$ & \\
\hline $1300-1500$ & $\mathrm{~N}_{2}$ & $a^{1} \Pi_{g}(L-B-H)$ & $7 k\{140\}$ & & & $\sim 100 \mathrm{k}$ \\
\hline 1304 & 0 & $3 s^{3} S^{\circ}(2 u v)$ & $7.5 k\{190\}$ & & & $25 k$ \\
\hline 1356 & 0 & $3 s^{5} s^{\circ}(1 u v)$ & $0.35 k\{140\}$ & & & $5 k$ \\
\hline 1493,1744 & $\mathrm{~N}$ & $3 s^{2} p(4 u v, 9 u v)$ & & & & $\sim 7.5 \mathrm{k}$ \\
\hline $1900-2300$ & NO & $A^{2} \Sigma^{+}(\gamma$ bands $)$ & $1 k[70-150]$ & & & \\
\hline $2000-4000$ & $\mathrm{~N}_{2}$ & $A^{3} \Sigma_{U}^{+}(V-K)$ & & & & $45 k$ (var.) \\
\hline $2600-3800$ & $\mathrm{O}_{2}$ & $A^{3} \Sigma_{u}^{+}($Herzberg I $)$ & & & $\approx 1.5 \mathrm{k}[90]$ & \\
\hline $2800-5000$ & $\mathrm{~N}_{2}$ & $C^{3} \Pi_{U}(2 P)$ & $0.9 k \quad[>130]$ & & & $90 k\left\{\begin{array}{l}(0,0) 03371 \\
25 k\end{array}\right.$ \\
\hline 2972 & 0 & ${ }^{1} S[2 F]$ & $0.15 \mathrm{k}$ & 20 & 12 & $5 k$ \\
\hline 3466 & $\mathrm{~N}$ & ${ }^{2} \mathrm{P}[2 \mathrm{~F}]$ & & & & $2.4 \mathrm{k}$ (var.) \\
\hline $3500-5500$ & $\mathrm{~N}_{2}^{+}$ & $B^{2} \Sigma_{U}^{+}(1 N)$ & $2 k[150]$ & $0.2-0.5[300]$ & $<1$ & $150 k$ \\
\hline 3727 & $0^{+}$ & ${ }^{2} D[I F] \& 3 p^{4} S^{\circ}(3)$ & & & & \\
\hline 3736 & $0^{+}$ & $3 p^{4} S^{\circ}(3)$ & & & & \\
\hline 3889 & $\mathrm{He}$ & $3 p^{3} p^{\circ}(2)$ & & $1[>400]$ & & \\
\hline 3914 & $\mathrm{~N}_{2}^{+}$ & $B^{2} \Sigma_{U}^{+}[1 N(0,0)]$ & & & & $100 k$ \\
\hline 3934 & $\mathrm{Ca}^{+}$ & $4 p^{2} p \rightarrow 4 s^{2} S$ & & & & \\
\hline 3947 & 0 & $4 p^{5} p(3)$ & & & & $\sim 0.1 \mathrm{k}$ \\
\hline 3967 & $0^{+}$ & $3 p^{2} p^{\circ}(6)$ & & & & \\
\hline 4041 & $\mathrm{~N}^{+}$ & $4 f^{3} G(39)$ & & & & $0.3 k$ \\
\hline 4076 & $0^{+}$ & $3 d^{4} F(10)$ & & & & $\sim 0.5 \mathrm{k}$ \\
\hline 4278 & $\mathrm{~N}_{2}^{+}$ & $B^{2} \Sigma_{U}^{+}[1 N(0,1)]$ & & & & $30 k$ \\
\hline 4340 & $\mathrm{H}$ & $5^{2}(S, P, D) \quad(H \gamma)$ & & & & \\
\hline 4368 & 0 & $4 p^{3} p(5)$ & & 1 & & $0.3 k$ \\
\hline 4418 & $0^{+}$ & $3 p^{2} D^{\circ}(5)$ & & & & $0.2 \mathrm{k}$ \\
\hline 4623 & $\mathrm{~N}^{+}$ & $3 p^{3} p(5)$ & & & & $0.3 k$ \\
\hline 4649 & $0^{+}$ & $3 p^{4} D^{\circ}(1)$ & & & & $\sim 0.5 \mathrm{k}$ \\
\hline 4861 & $\mathrm{H}$ & $4^{2}(S, P, D)\left(H_{\beta}\right)$ & & & $1\{200\}$ & $\sim 0.1 \mathrm{k}$ ( $\mathrm{H}$ aurora) \\
\hline $5000-6500$ & $\mathrm{NO}_{2}$ & continuum & & & $\approx 1$ per $\AA$ & \\
\hline $5000-9000$ & $0_{2}^{+}$ & $b^{4} \Sigma \bar{g}(1 N)$ & & & & $26 k\left\{\begin{array}{l}(1,0) @ 5609 \\
\approx 6 k\end{array}\right.$ \\
\hline 5005 & $\mathrm{~N}^{+}$ & $3 d^{3} F^{\circ}(19)$ & & & & $0.6 \mathrm{k}$ \\
\hline $0.505-\approx 2.5 \mu \mathrm{m}$ & $\mathrm{N}_{2}^{+}$ & $A^{2} \Pi_{U}($ Meinel $)$ & $10 k$ & & & $600 \mathrm{k}\left\{\begin{array}{l}(0,0) 01.11 \mu \mathrm{m} \\
110 \mathrm{k}\end{array}\right.$ \\
\hline 5200 & $\mathrm{~N}^{2}$ & ${ }^{2} D^{\circ}[\mathrm{FF}]$ & $90[200-250]$ & 10 & $\approx 1$ & $0.3 k$ \\
\hline $5300-6300$ & $\mathrm{OH}$ & $\chi^{2} \Pi(\Delta v=6$ seq. $)$ & & & $0.2 k[85]$ & \\
\hline
\end{tabular}

60 /SPIE Vol. 91 Methods for Atmospheric Radiometry (1976) 
OPTICAL RADIATION FROM THE ATMOSPHERE

Table 1. (cont.)

\begin{tabular}{|c|c|c|c|c|c|c|}
\hline \multirow{2}{*}{$\begin{array}{l}\text { Wavelength } \\
(\AA \text { or } \mu \mathrm{m})\end{array}$} & \multirow{2}{*}{ Species } & \multirow{2}{*}{ Identification ${ }^{b}$} & \multicolumn{4}{|c|}{ Rayleigh Radiance (R) in the Zenith } \\
\hline & & & Day & TwiTight & Night & IBC III Aurora \\
\hline $0.53-\approx 7 \mu \mathrm{m}$ & $\mathrm{N}_{2}$ & $B^{3} \Pi_{g}(1 P)$ & $10 k[150]$ & & $00 \mathrm{k} / 35 \mathrm{k}$ & \\
\hline 5577 & 0 & ${ }^{1} S[3 F]$ & $3 k[90-300]$ & $0.4 \mathrm{k}$ & $0.25 \mathrm{k}$ & $100 \mathrm{k}$ \\
\hline 5679 & $\mathrm{~N}^{+}$ & $3 p^{3} D(3)$ & & & & $0.7 k$ \\
\hline 5876 & $\mathrm{He}$ & $3 d^{3} D(11)$ & & & & \\
\hline $5890-6$ & $\mathrm{Na}$ & $3 p^{2} p \rightarrow 3 s^{2} s(D-1$ ines $)$ & $30 \mathrm{k}[92]$ & $1-4 k \quad[92]$ & $0.02-0.15[92]$ & $\approx 1 \mathrm{k}$ \\
\hline 6157 & 0 & $4 d^{5} D^{\circ}(10)$ & & & & $\sim 0.1$ \\
\hline $6200-7700$ & $\mathrm{OH}$ & $x^{2} \Pi(\Delta v=5$ seq. $)$ & & & $2 k[85]$ & \\
\hline $6300-64$ & 0 & ${ }^{1} \mathrm{D}[\mathrm{IF}]$ & $2-20 k[250-300]$ & $1 \mathrm{k}$ & $0.01-0.5 k$ & $7 k$ \\
\hline $6350-9000$ & $\mathrm{O}_{2}$ & $b^{1} \Sigma_{g}^{+}(A t)$ & $0.3 M[40-120]$ & & $\sim 1 \mathrm{k}$ & $1.3 \mathrm{M}$ \\
\hline 6563 & $\mathrm{H}$ & $3^{2}(S, P, D)\left(H_{\alpha}\right)$ & & & $3[200]$ & $\sim 0.3 \mathrm{k}$ \\
\hline 6708 & $\mathrm{Li}$ & $2 p^{2} p^{\circ}(1)$ & & $0.01-1 k[90]$ & $0.20 \mathrm{k}[90]$ & \\
\hline $7319-30$ & $0^{+}$ & ${ }^{2} \mathrm{p}^{\circ}[2 \mathrm{~F}]$ & $<1 \mathrm{k}$ & & & 0.4 \\
\hline $0.75-1.01 \mu \mathrm{m}$ & $\mathrm{OH}$ & $x^{2} \Pi(\Delta v=4)$ & & & $12 k[85]$ & \\
\hline 7619 & $\mathrm{O}_{2}$ & $\mathrm{~b}^{1} \Sigma_{\mathrm{g}}^{+}[\operatorname{At}(0,0)]$ & $300 k[40-120]$ & & $6 k$ & $\sim 7 M$ \\
\hline 7699 & K & $4 p^{2} p \rightarrow 4 s^{2} S$ & & 40 & & \\
\hline 7774 & 0 & $3 p^{5} p(1)$ & $\sim 1.6 \mathrm{k}$ & & $\sim 0.2 k$ & $7 k$ \\
\hline 8212 & $N$ & $3 p^{4} p^{\circ}(2)$ & . & & & $3 k$ \\
\hline 8447 & 0 & $3 p^{3} p(4)$ & $\sim 1.1 \mathrm{k}$ & & & $15 k$ \\
\hline 8618 & $\mathrm{~N}$ & $3 p^{2} p^{\circ}(8)$ & & & & $4 k$ \\
\hline 8645 & $\mathrm{O}_{2}$ & $\mathrm{~b}^{1} \Sigma_{\mathrm{g}}^{+}[\mathrm{At}(0,1)]$ & $10 k[40-120]$ & & $1 k[80]$ & $60 k$ \\
\hline 8692 & $\mathrm{~N}$ & $3 p^{4} D^{\circ}(1)$ & & & & $11 \mathrm{k}$ \\
\hline 9400 & $\mathrm{H}_{2} \mathrm{O}$ & $(201) B_{1}$ & & & & \\
\hline $0.98-1.4 \mu \mathrm{m}$ & $\mathrm{OH}$ & $x^{2} \Pi(\Delta v=3)$ & & & $90 k$ [85] & \\
\hline$\sim 1$ & $\mathrm{NO}_{2}$ & continuum & $10 \mathrm{M}$ & & & \\
\hline 1.04 & $\mathrm{~N}$ & ${ }^{2} P^{\circ}[3 F]$ & & & & $35 k$ (est.) \\
\hline $1.06-1.60$ & $\mathrm{O}_{2}$ & $a^{1} \Delta_{g}($ IR At $)$ & $20 \mathrm{M}[60-70]$ & $5 M$ & $\approx 85 \mathrm{k}[90 \mathrm{~km}]$ & $\sim 1 M$ (var.) \\
\hline 1.083 & $\mathrm{He}$ & $2 p^{3} p^{\circ}(1)$ & & $0.1-3 k[500]$ & & \\
\hline 1.129 & 0 & $3 d^{3} D^{\circ}$ & & & & \\
\hline 1.130 & 0 & $4 s^{5} S^{\circ}(7)$ & & & & \\
\hline 1.14 & $\mathrm{H}_{2} \mathrm{O}$ & $(111) B_{1}$ & & & & \\
\hline 1.27 & $\mathrm{O}_{2}$ & $a^{1} \Delta_{g}[\operatorname{IR~At~}(0,0)]$ & $\approx 20 \mathrm{M}$ & $\approx 5 M$ & $\approx 80 \mathrm{k}$ & $\sim 1 M$ (var.) \\
\hline 1.316 & 0 & $4 s^{3} S^{0} \rightarrow 3 p^{3} p$ & & & & $2 k$ \\
\hline 1.38 & $\mathrm{H}_{2} \mathrm{O}$ & $(101) B_{1}$ & & & & \\
\hline $1.4-2.3$ & $\mathrm{OH}$ & $x^{2} \Pi(\Delta v=2)$ & & & $0.55 \mathrm{M}$ & \\
\hline 1.58 & $\mathrm{O}_{2}$ & $\mathrm{a}^{1} \Delta_{\mathrm{g}}[\operatorname{IR}$ At $(0,1)]$ & $300 \mathrm{k}[60-70]$ & $80 k$ & $4 k$ & \\
\hline 1.88 & $\mathrm{H}_{2} \mathrm{O}$ & $(001) B_{1}$ & & & & \\
\hline 2.0 & $\mathrm{CO}_{2}$ & $\left(12^{\circ} 1\right)$ & & & & \\
\hline $2.65-2.80$ & $\mathrm{H}_{2} \mathrm{O}$ & $(100) A_{1} \&(100) B_{1}$ & & & $35 k\{9\}$ & \\
\hline $2.7-3.5$ & NO & $x^{2} \Pi(\Delta v=2)$ & & & & \\
\hline $2.8-4.5$ & $\mathrm{OH}$ & $x^{2} \Pi(\Delta v=1)$ & & & $0.35 \mathrm{M}[85]$ & \\
\hline 3.3 & $\mathrm{CH}_{4}$ & $(00011001)\left(v_{3}\left(f_{2}\right)\right)$ & & & I2M [85] & \\
\hline 4.3 & $\mathrm{CO}_{2}$ & $\left(00^{\circ} 1\right)\left(v_{3}\right)$ & & & $1160 \mathrm{M}[12]$ & \\
\hline 4.5 & $\mathrm{~N}_{2} \mathrm{O}$ & $\left(00^{\circ} 1\right)\left(v_{3}\right)$ & & & & \\
\hline 4.8 & $\mathrm{CO}_{2}$ & $\left(11^{1} 0\right)$ & & & $0.2 \mathrm{M}[85]$ & \\
\hline $4.9-6.0$ & NO & $\mathrm{X}^{2} \Pi(\Delta v=1)$ & & & $4.7 M\{84\}$ & \\
\hline
\end{tabular}


DORAN J. BAKER, WILLIAM R. PENDLETON, JR.

Table 1. (cont.)

\begin{tabular}{|c|c|c|c|c|c|c|}
\hline \multirow{2}{*}{$\begin{array}{l}\text { Wavelength } \\
(\AA \text { or } \mu \mathrm{m})\end{array}$} & \multirow{2}{*}{ Species } & \multirow{2}{*}{ Identification ${ }^{b}$} & \multicolumn{4}{|c|}{ Rayleigh Radiance (R) in the Zenith } \\
\hline & & & Day & Twilight & Night & IBC III Aurora \\
\hline $6.2 \mu \mathrm{m}$ & $\mathrm{NO}_{2}$ & $v_{3}\left(b_{1}\right)$ & & & & \\
\hline 6.3 & $\mathrm{H}_{2} \mathrm{O}$ & $(010) A_{1}$ & & & $1800 \mathrm{M}[12]$ & \\
\hline 7.6 & $\mathrm{CH}_{4}$ & $(00000111)\left(v_{4}\left(f_{2}\right)\right)$ & & & & \\
\hline 7.8 & $\mathrm{~N}_{2} \mathrm{O}$ & $\left(10^{\circ} 0\right)\left(\nu_{1}\right)$ & & & $2500 M[12]$ & \\
\hline 9.4 & $\mathrm{CO}_{2}$ & $\left(00^{\circ} 1\right)-\left(02^{\circ} 0\right)$ & & & & \\
\hline 9.6 & $0_{3}$ & $(001)\left(v_{3}\left(b_{1}\right)\right)$ & & & $10 \mathrm{M}\{80\}$ & $16 M(I I)$ \\
\hline 10.3 & $\mathrm{CO}_{2}$ & $\left(00^{\circ} 1\right)-\left(10^{\circ} 0\right)$ & & & $19 k\{80\}$ & \\
\hline 11.3 & $\mathrm{HNO}_{3}$ & & & & $1500 \mathrm{M}\{8\}$ & \\
\hline 13.8 & $\mathrm{CO}_{2}$ & $\left(10^{\circ} 0\right)-\left(01^{1} 0\right)$ & & & $0.35 \mathrm{M}\{80\}$ & $0.7 \mathrm{M}$ (II) \\
\hline 15.0 & $\mathrm{CO}_{2}$ & $\left(01^{I} 0\right)\left(\nu_{2}\right)$ & & & $\begin{array}{l}\begin{array}{l}237 M\{80\} \\
\{42,000 M\{8\}\end{array}\end{array}$ & \\
\hline 17.0 & $\mathrm{~N}_{2} \mathrm{O}$ & $\left(01^{I} 0\right)\left(\nu_{2}\right)$ & & & $0.34 M\{80\}$ & \\
\hline 19.2 & $\mathrm{H}_{2} \mathrm{O}$ & rotational & & & $7 k\{7\}$ & \\
\hline 63 & 0 & ${ }^{3} p_{2}-{ }^{3} p_{1}$ & & & $1.7 M\{120\}$ & \\
\hline 147 & 0 & ${ }^{3} P_{1}-{ }^{3} P_{0}$ & & & & \\
\hline
\end{tabular}

(a) Wavelengths given for atomic features are commonly-used values for the multiplets. The molecular-band wavelengths are also commonly-used values. Spectral intervals are indicated for band systems.

(b) Identifications:

(1) The designation of the upper state for a transition is followed by the multiplet number (in parentheses), or other suitable designation of the transition. The multiplet numbers follow the widely-adopted assignments of Mrs. Moore-sitterly in her NBS compilation of spectral data. In cases where the transition is not indicated, it may be assumed that the lower level is the ground state.

(2) Key to abbreviations:

1 uv $\equiv$ ultraviolet multiplet No. 1 in NBS compilations.

$\left(L_{\alpha}, L_{\beta}\right) \equiv$ Lyman alpha and beta emissions of atomic hydrogen.

L-B-H E Lyman-Birge-Hopfield $(a \rightarrow X)$ band system of $\mathrm{N}_{2}$.

$V-K \equiv$ Vegard-Kaplan $(A \rightarrow X)$ band system of $N_{2}$.

$\gamma$-bands $\equiv$ Gamma band system $(A \rightarrow X)$ of NO.

Herzberg $\mathrm{I} \equiv A \rightarrow X$ system of $\mathrm{O}_{2}$.

$1 P \equiv$ First positive system $(B \rightarrow A)$ of $\mathrm{N}_{2}$.

$2 P \equiv$ Second positive system $(C \rightarrow B)$ of $N_{2}$.

IF E Forbidden multiplet No. 1 in NBS compilations.

IN $\equiv$ First negative system of $N_{2}^{+}(B \rightarrow X)$ and $0_{2}^{+}(b \rightarrow a)$.

$\left(H_{0}, H_{B}\right) \equiv$ Balmer alpha and beta emissions of atomic hydrogen.

Meinel $\equiv$ Meinel band system $(A \rightarrow X)$ of $\mathrm{N}_{2}^{+}$.

At $\equiv$ Atmospheric system $(b \rightarrow X)$ of $0_{2}$.

IR At $\equiv$ Infrared atmospheric system $(a \rightarrow X)$ of $\mathrm{O}_{2}$.

(c) 5-10 \{750\} indicates a zenith intensity of 5-10 R from an observing altitude of $750 \mathrm{~km}$. An entry such as 1k [70-150] indicates a total zenith intensity of 1000R resulting from an emitting region predominantly between 70 and $150 \mathrm{~km}$. An entry such as 2k [85] indicates a total zenith intensity associated with an emitting region having a characteristic altitude of $85 \mathrm{~km}$.

(d) A number of the entries in this table are from Hunten. ${ }^{14}$ 